\title{
Lack of cardiovascular risk assessment in inflammatory arthritis and systemic lupus erythematosus patients at a tertiary care center
}

\author{
Stephanie O. Keeling $\cdot$ Michelle Teo $\cdot$ Daisy Fung
}

Received: 22 February 2011 /Revised: 29 March 2011 /Accepted: 31 March 2011 / Published online: 19 April 2011

(C) Clinical Rheumatology 2011

\begin{abstract}
The purpose of this study is to evaluate cardiovascular risk assessment at a Canadian rheumatology center and describe the cardiovascular risk of inflammatory arthritis (IA) and systemic lupus erythematosus (SLE) patients using the Framingham risk score. A retrospective chart review of 504 patients attending nine rheumatology practices at the University of Alberta Hospital was performed. A pre-specified case report form detailed patient demographics, cardiac risk factors, variables for the Framingham 2008 score, disease activity, and medication use. In this group of 504 patients, $64(12.7 \%)$ had SLE (male (M) to female $(\mathrm{F})$ ratio $=60: 4)$ and $440(87.3 \%)$ had an IA (M to F ratio=117:323). Of the SLE patients, $31(48.4 \%)$ met four or more American College of Rheumatology (ACR) criteria, 33 (51.6\%) had less than four ACR criteria. Of the IA patients, 156 (35.5\%) were CCP positive and 257 (58.4\%) were RF positive. Utilizing the chart data, retrospective Framingham risk scores were calculable for one (1.6\%) SLE patient and three $(0.68 \%)$ IA patients. The most common cardiac risk factors not documented in the medical records of both the SLE and IA patients included: (1) positive family history of MI, (2) diabetes, and (3) lipid status. The blood pressure was more frequently documented in the SLE patients
\end{abstract}

\footnotetext{
S. O. Keeling $\cdot$ M. Teo

Division of Rheumatology, Department of Medicine,

University of Alberta,

Edmonton, AB, Canada

S. O. Keeling $(\bowtie)$

562 Heritage Medical Research Center, University of Alberta,

Edmonton, AB T6G 2S2, Canada

e-mail: stephanie.keeling@ualberta.ca

D. Fung

Department of Family Medicine,

University of Alberta,

Edmonton, AB, Canada
}

(93.8\%) compared to the IA patients (56.1\%). While traditional cardiac risk factors only partially contribute to the increased cardiovascular risk in these patients, cardiovascular risk assessment was suboptimally performed amongst a large group of rheumatologists. A dedicated cardiovascular risk reduction clinic for inflammatory rheumatic diseases has been established at this site to fulfill this need and evaluate treatment strategies.

Keywords Cardiovascular risk reduction $\cdot$ Framingham risk score $\cdot$ Inflammatory arthritis $\cdot$ Systemic lupus erythematosus

\section{Introduction}

An increased risk of cardiovascular disease (CVD) in patients with rheumatic diseases including inflammatory arthritis (IA) and systemic lupus erythematosus (SLE) is well recognized [1-3]. While IA patients are treated with disease modifying anti-rheumatic drugs (DMARDs) and biologic agents that reduce disease activity, multiple studies have demonstrated increased cardiovascular (CV) risk despite conventional anti-rheumatic therapy [4-7]. Several studies of rheumatoid arthritis (RA) patients with disease durations of less than 5 years demonstrated an increased $\mathrm{CV}$ risk, suggesting this excess risk is an early feature of RA [4, 8-11]. The risk appears to accumulate over time and persists after adjustment for other cardiovascular risk factors (relative risk of MI is greater than three after 10 years of having the disease) $[6,12]$ suggesting that RA independently doubles the hazard for myocardial infarction and sudden cardiac death. The degree of increased risk of myocardial infarction in SLE patients varies, with risks as high as 50 -fold in premenopausal women and decreasing to the risk of the general population in the elderly [13-15]. The 
nurses' health study found a slightly greater than twofold risk of cardiovascular disease in lupus patients [16].

Reasons for the increased risk of CVD in these inflammatory rheumatic disease patients include the co-existence of traditional cardiac risk factors, including diabetes, hypertension, and hypercholesterolemia; however, the additional factor of inflammation and endothelial dysfunction are also felt to be an important contributors to this risk [2, 17]. Abnormal lipid profiles, specifically reduced high-density lipoproteins (HDLs) and elevated triglycerides have been described in these populations $[2,17]$.

The severity of inflammatory activity predicts CV events and mortality in RA patients $[6,18,19]$. Certain medications used to treat IA and SLE may also contribute to the increased risk of CVD, including nonsteroidal anti-inflammatory drugs (NSAIDs) [20], COX-2 inhibitors [5, 20], steroids [21, 22], and anti-folate medications such as methotrexate [23, 24].

Given the mixed picture of contributors to the increased CV risk in SLE and IA patients, an active risk assessment and management is entirely appropriate in this population. The European League Against Rheumatism (EULAR) has already reflected this in their recent recommendations put forth by two different panels suggesting annual $\mathrm{CV}$ risk assessment for both RA patients and SLE patients respectively $[25,26]$.

Given the importance of CV risk assessment in inflammatory rheumatic diseases, the main objective of this study was to evaluate the extent of $\mathrm{CV}$ risk assessment in a university rheumatology center and determine the CV risk of SLE and IA patients followed by a diverse group of practicing rheumatologists. The main hypotheses included that (1) CV risk assessment and, therefore, management would be limited in the typical rheumatology practice and that (2) CV risk would be increased with intermediate to high Framingham risk scores for those SLE and IA patients where data were collected.

\section{Methods}

A retrospective chart review of nine rheumatologists at the University of Alberta was performed after obtaining a university ethics approval to access patient paper charts. A pre-specified case report form was created for the study to standardize data collection per chart. Data collection included cardiovascular-specific data and IA/SLE disease-specific data. The cardiac risk factors were recorded in a dichotomous format (i.e., "yes" or "no"); however, in instances where no data was present in the chart, a third option "not recorded" was chosen to account for the fact that many patients did not have the variable assessed, and one could not assume this meant they were lacking a particular risk factor.

The data collection included: patient demographics without personal identifiers, traditional cardiac risk factors included in the Framingham 10-year risk for general cardiovascular disease [27] (age, gender, systolic hypertension, family history, smoking, diabetes, dyslipidemia), obesity, cardiac medications (acetylsalicylic acid (ASA), anti-cholesterol agents, anti-hypertensives), history of cardiac evaluations, IA/SLE disease duration, normal versus abnormal CRP (normal, $<8 \mathrm{mg} / \mathrm{L}$ ) and ESR (normal, $<20 \mathrm{~mm} / \mathrm{h}$ ), seropositivity (rheumatoid factor and anti-cyclic citrullinated peptide), number of swollen or tender joints, number of American College of Rheumatology (ACR) criteria met for SLE [28], anti-nuclear antibody (ANA), ds-DNA and extractable nuclear antigen (ENA) positivity, IA/SLE medication history, history of cardiac events under care of rheumatologist, and recorded blood pressures in the chart.

The presence of hypertension was recorded as "yes" only when the specific diagnosis was recorded in the chart and/or the patient was taking an anti-hypertensive medication. Diabetes was recorded as "yes" when documented by the rheumatologist or referring physician or confirmed by the presence of diabetes-related treatment(s). At the time of the review, there were no guidelines for lipid targets for inflammatory arthritis patients; therefore, we classified the patients as medium risk for CVD according to the Canadian 2009 dyslipidemia guidelines [29], therefore documenting dyslipidemia according to the following: the patient was on an anti-cholesterol medication and/or low-density lipoprotein (LDL) was equal to or greater than $3.5 \mathrm{mmol} / \mathrm{L}$ and/or the total cholesterol to HDL was equal to or greater than 5 . Obesity was determined by the rheumatologists' documentation. Cardiac medication history involved the use of ASA, anti-cholesterol, or anti-hypertensive drugs. Rheumatologistinitiated referrals for cardiovascular risk reduction, including referrals for further cardiac investigations, specialist(s), or initiating cardiac medications were recorded. Any CV events (including myocardial infarction, cerebrovascular accident, congestive heart failure) occurring under the care of a rheumatologist in the chart were documented. The Framingham risk scores were calculated using an online calculator at http://www.framinghamheartstudy.org/risk/gencardio.html.

IA patients included those with seropositive or negative RA and psoriatic arthritis. For both IA and SLE patients, the diagnosis relied entirely on the rheumatologist's assessment and chart documentation rather than specific ACR criteria. Patients diagnosed with SLE by the rheumatologist were included (even if they did not meet the minimum four ACR classification criteria for SLE [28]) if the chart documentation was clearly SLE as defined by biopsy (e.g., renal) and/or there was no documentation of debate about the diagnosis or alternative diagnoses for the SLE features recorded in the chart. Any patient with a diagnosis including overlap syndrome, mixed connective tissue disease, undifferentiated connective tissue disease was excluded. Patients with early, undeclared inflammatory rheumatic diseases were excluded. All IA and SLE charts 
Table 1 Systemic lupus erythematosus patient characteristics

\begin{tabular}{lc}
\hline Characteristic & No. (\%) of patients \\
\hline Number of patients with SLE & $64(12.7)$ \\
Male to female ratio & $4(6.2): 60(90.4)$ \\
Meeting 4 or more ACR classification criteria for SLE & $31(48.4)$ \\
Anti-nuclear antibody positive & $43(67.2)$ \\
Double-stranded DNA positive & $6(9.4)$ \\
Extractable nuclear antigen positive & \\
(1 or more of: SSA, SSB, anti-RNP, anti-Smith) & $21(32.8)$ \\
Malar rash & $13(20.3)$ \\
Discoid rash & $6(9.4)$ \\
Photosensitivity & $9(14.0)$ \\
Oral ulcers & $14(21.9)$ \\
Arthritis & $31(48.4)$ \\
Serositis & $10(15.6)$ \\
Renal & $27(42.2)$ \\
Neurologic & $3(4.7)$ \\
Anemia (Hb $<120$ g/L) & $19(29.7)$ \\
Leukopenia $(W B C<4.0)$ & $15(23.4)$ \\
Thrombocytopenia (platelets $<150)$ & $6(9.4)$ \\
Lupus anticoagulant positivity & $7(10.9)$ \\
Anticardiolipin positivity & $5(7.8)$ \\
\hline
\end{tabular}

available at the time of data collection were included in the study. Data were entered into a Microsoft access database. Descriptive statistics including means and standard deviations were calculated using Microsoft excel.

\section{Results}

A total of 504 patient charts, 64 with SLE and 440 with IA were reviewed. The characteristics of SLE and IA patients are displayed in Tables 1 and 2, respectively. The mean disease duration for SLE patients was 10.3 years $( \pm 8.2)$, and the mean age at diagnosis was 29.9 years $( \pm 15.3)$. Slightly less than $50 \%$ of the SLE patients met four or more ACR classification criteria for SLE [28], the most common manifestations included arthritis (31 patients), renal (27 patients), and oral ulcers (14 patients). Twelve SLE patients were ANA negative, and further evaluation confirmed that six of these patients were also ENA negative; one patient's ENA was not available and the remaining five patients were ENA positive.

Of the 440 IA patients, 363 were diagnosed as seropositive RA, 77 as seronegative RA, and seven as having psoriatic arthritis. The mean number of swollen and tender joints at first rheumatology visit were 8.6 $( \pm 6.4)$ and $11.7( \pm 9.1)$, respectively. Few extra-articular manifestations of IA were noted, the most common being nodulosis. The mean age at diagnosis was 45 years $( \pm 16.1)$, and the mean disease duration was 11.9 years $( \pm 11.5)$. Abnormal CRP (>8 mg/L) was noted in $19(30 \%)$ of SLE and 259 (58.9\%) of IA patients, whereas abnormal ESR $(>20 \mathrm{~mm} / \mathrm{h})$ was noted in $27(42 \%)$ of SLE and 282 $(64.1 \%)$ of IA patients.
Table 2 Inflammatory arthritis patient characteristics

\footnotetext{
${ }^{a}$ Rheumatoid factor and anticyclic citrullinated peptide status were not available in the chart for 76 and 207 patients, respectively
}

\begin{tabular}{lc}
\hline Characteristics & No. (\%) of patients \\
\hline Number of patients with inflammatory arthritis Male to female ratio & $440(83.7)$ \\
Rheumatoid factor positive $^{\mathrm{a}}$ & $117(26): 323(73.4)$ \\
Rheumatoid factor negative $^{\text {Anti-cyclic citrullinated peptide positive }^{\mathrm{a}}}$ & $257(58.4)$ \\
Anti-cyclic citrullinated peptide negative $^{\text {Seropositive rheumatoid arthritis (as defined by rheumatologist in chart) }}$ & $107(24.3)$ \\
Rheumatoid pulmonary disease $^{\text {Rheumatoid nodulosis }}$ & $156(35.5)$ \\
\hline
\end{tabular}


Table 3 Medication use in the SLE and IA patients

\begin{tabular}{lcc}
\hline Medication & $\begin{array}{l}\text { No. (\%) of SLE patients with past or current medication } \\
\text { exposure }\end{array}$ & $\begin{array}{l}\text { No. (\%) of IA patients with past or current medication } \\
\text { exposure }\end{array}$ \\
\hline Prednisone & $38(59.4)$ & $62(14.1)$ \\
NSAIDs and COX-2 & $7(6.3)$ & $154(35)$ \\
$\quad$ inhibitors & $11(17.2)$ & $300(68.6)$ \\
Methotrexate & $58(90.6)$ & $276(68.1)$ \\
Antimalarials & $1(1.6)$ & $88(20)$ \\
Sulfasalazine & $1(1.6)$ & $112(22.5)$ \\
Leflunomide & $1(1.6)$ & $49(11.1)$ \\
Gold (oral/injectable) & 0 & $49(11.1)$ \\
Infliximab & 0 & $28(6.3)$ \\
Adalimumab & 0 & $64(14.5)$ \\
Etanercept & 0 & $9(2)$ \\
Abatacept & $2(3.1)$ & $9(2)$ \\
Rituximab & $15(23.4)$ & 0 \\
Azathioprine & $17(26.6)$ & 0 \\
Mycophenolate mofetil & & \\
\hline
\end{tabular}

Current or past medication use for SLE and IA disease activity is represented in Table 3. A larger percentage of SLE patients had past or present exposure to prednisone for their disease than IA patients (59\% vs 14\%), whereas more IA patients had past or present exposure to NSAIDs than SLE patients (35\% vs $6 \%$ ). More than $90 \%$ of SLE patients had exposure to antimalarials at some point in their disease. Exposure to the three available TNF inhibitors ranged from $5 \%$ to $15 \%$ of IA patients.

The most common cardiac risk factors not documented in the patient record included family history of premature coronary artery disease, diabetes, and obesity (Table 4). Recording of classically recognized cardiac medications including ASA, anti-hypertensives, and lipid-lowering agents was done less frequently in IA patients compared to SLE patients (Table 5). The use of lipid-lowering agents in both populations of patients was low compared to antihypertensive agents, with a greater percentage of SLE patients on anti-hypertensive agents (39\% of SLE vs $18.2 \%$ of IA patients).

The most prevalent cardiac risk factor (when recorded) for SLE and IA patients was hypertension followed by dyslipidemia (Fig. 1). Systolic blood pressures were documented in $60(93.8 \%)$ of SLE patients compared with $247(56.1 \%)$ of IA patients.

The Framingham risk score for a 10-year risk of general cardiovascular disease [27] was calculable for one SLE patient and three IA patients. The Framingham risk for any cardiovascular event in the next 10 years for the three IA patients was $48 \%, 21 \%$, and $17 \%$, respectively and for the SLE patient was $20 \%$. The risk for all these patients would be classified as high except for one patient who would moderately be at risk (17\%). Cardiovascular events were recorded in two IA patients, and rheumatologists ordered cardiovascular evaluations in ten patients.

\section{Discussion}

This descriptive study effectively described the extent to which a large number of rheumatologists at one tertiary care center are not performing cardiovascular risk assessments for their IA and SLE patients. The study could not determine the cardiac risk of the majority of both SLE and IA patients as records did not include their traditional cardiac risk factors, and Framingham risk scores were,
Table 4 Number (\%) of SLE and IA patients where traditional cardiovascular risk factors were not recorded

\begin{tabular}{lcc}
\hline Cardiac risk factor & No. (\%) of SLE patients & No. (\%) of IA patients \\
\hline Smoking & $33(51.6)$ & $261(59.3)$ \\
Dyslipidemia & $58(58)$ & $377(85.7)$ \\
Hypertension & $2(3.1)$ & $123(28)$ \\
Diabetes mellitus & $62(96.9)$ & $421(95.7)$ \\
Family history of premature coronary artery disease & $61(95.3)$ & $433(98.4)$ \\
Obesity & $59(92.9)$ & $422(96)$ \\
\hline
\end{tabular}


Table 5 Number (\%) of SLE and IA patients taking cardiovascular medications when recorded

$N R$ not recorded in chart

\begin{tabular}{llcc}
\hline Medication & ASA & Anti-hypertensives & Lipid-lowering agents \\
\hline SLE patients & $7(10.9)$ & $25(39.1)$ & $8(12.5)$ \\
& $(\mathrm{NR}=2$ patients $)$ & $(\mathrm{NR}=2$ patients $)$ & $(\mathrm{NR}=3$ patients $)$ \\
IA patients & $34(7.7)$ & $80(18.2)$ & $41(9.3)$ \\
& $(\mathrm{NR}=127$ patients $)$ & $(\mathrm{NR}=123$ patients $)$ & $(\mathrm{NR}=130$ patients $)$ \\
\hline
\end{tabular}

therefore, not calculable. Other existing risk scores were also not calculable due to the lack of documentation of traditional cardiovascular risk factors. The cardiac medications were poorly documented in the IA patients as compared to the SLE patients. Possible explanations for the discrepancy include the greater potential for comprehensive, multiphysician care in some SLE compared to IA patients, including those SLE patients with renal disease requiring ACE inhibition for renal protection.

For the four patients with calculable Framingham risks, three patients fell in the high-risk category where more aggressive cardiovascular risk reduction might serve useful, such as significant LDL lowering. However, the lipid status was not available on over half of the SLE and IA patients in the medical record suggesting this was not being assessed and treated. Hypertension was the most common traditional risk factor for both the SLE and IA patients. Possible

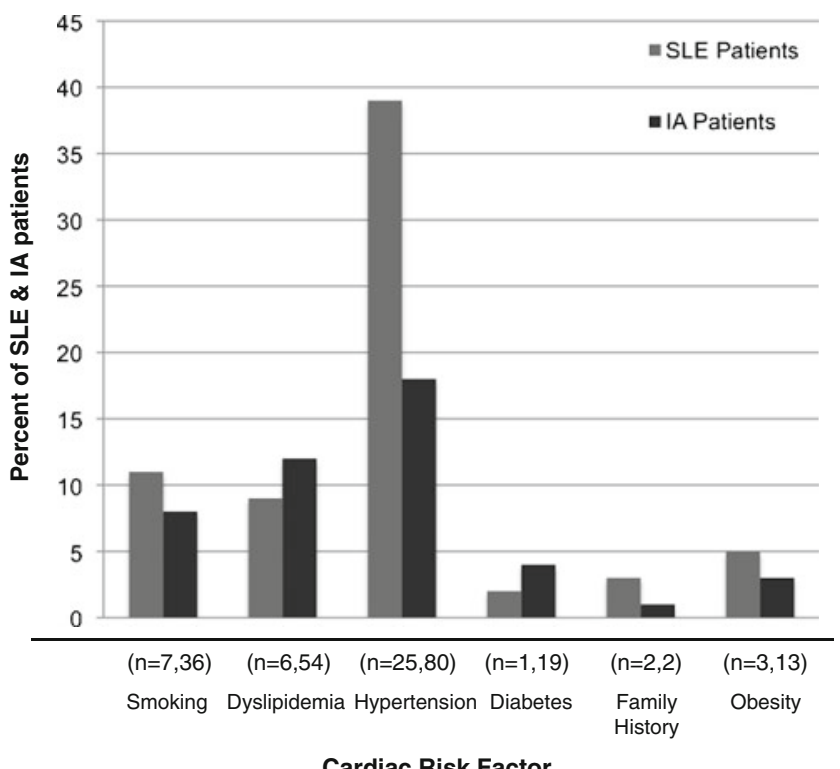

Cardiac Risk Factor

Fig. 1 Percentage of cardiac risk factors in SLE and IA patients where risk assessment was performed. The percentage of SLE and IA patients with recorded traditional cardiac risk factors are displayed. The actual number of SLE and IA patients respectively with each risk factor is displayed as ( $n=$ no. of SLE patients, no. of IA patients) along the $x$-axis. As reflected in Table 4, a significant number of SLE and IA patients did not have their traditional cardiac risk factors recorded, resulting in a low denominator of patients with reportable data. Obesity is not included in the Framingham risk score but is included due to its relative importance in cardiovascular risk assessment explanations include the influence of medications including prednisone, NSAIDs, and leflunomide as well as disease activity (e.g., renal lupus) on blood pressure. Despite better documentation in medication use in SLE patients, their traditional cardiac risk factor evaluation was no better than the IA patient, as reflected by only one lupus patient with a calculable Framingham risk score in the whole study. The number of IA patients on steroids at any point in their disease (14\%) may seem lower than expected compared to other sites but reflects the practice pattern adopted by the majority of local rheumatologists of limited steroid use in IA patients.

While this study is entirely descriptive and retrospective, a large number of rheumatology practices were audited, representing a broad range of clinical experience. The lack of risk assessment was common amongst all the rheumatologists, making it impossible to evaluate possible confounders in rheumatologists performing $\mathrm{CV}$ risk assessment, such as time from training (less than 1 year to greater than 35 years) and potential site and training bias. The sites involved in collecting prospective patient data or participating in many clinical trials may be more likely to evaluate traditional cardiac risk factors as part of their standard of care assessment. These sites would likely have more complete data and be able to risk stratify their patients. Interestingly, while many studies and clinical trials are performed at our site on an ongoing basis, the cardiovascular risk information was still lacking. The evaluation of the practice patterns of other tertiary and non-tertiary sites would be valuable to determine how extensive cardiac risk assessment is in the majority of rheumatologists' offices.

This retrospective chart review has some important limitations to acknowledge. For example, author interpretation of patient records may have led to underestimation of possible risks such as obesity (e.g., where no BMI was measured) or diabetes. The possibility that this information was available elsewhere was unlikely, as all rheumatologists at this site were functioning with paper charts at that time. Since approximately $50 \%$ of SLE patients did not meet the ACR SLE criteria [28], it is possible that some patients did not truly have a diagnosis of SLE despite measures outlined in the methods to limit this possibility. Early inflammatory arthritis patients without a definite diagnosis of rheumatoid or psoriatic arthritis would have been excluded, potentially leaving out an important population of patients to study in order to understand their CV risk. 
The current lack of active cardiovascular risk reduction at our site reflects several important possibilities. Until 2009, no guidelines existed on how to evaluate CV risk in patients with inflammatory arthritis. The chart review, which was performed in this period, reflected the practice patterns of rheumatologists up to 2009. The EULAR guidelines for cardiac risk assessment in RA patients, however, have not been rigorously tested in a prospective, multicentered study to determine their utility in cardiovascular risk stratification [25]. Moreover, uptake of any guidelines in clinical practice will be variable amongst practicing rheumatologists. The aggressiveness to which the rheumatologist should pursue aspects of risk reduction, including lipid lowering, blood pressure control, smoking cessation, and weight reduction, remains in debate. Presently, calculating a risk score is not as routine as calculating a disease activity score for a joint assessment in an IA patient or an SLE disease activity index in an SLE patient.

Other possible explanations as to why cardiovascular risk reduction is not performed at this site to a satisfactory level include that many rheumatologists may assume this task should be the sole responsibility of the primary care physician. This assumption is problematic, however, because many primary care physicians are not aware of the increased CV risk in SLE and IA patients and also how to treat them [30]. The lack of CV risk assessment is pervasive as it was evident in two distinct rheumatology populations at this site - the SLE and IA patients.

The Framingham risk score may not be the best option for the risk assessment of these patient populations as it may not be sensitive enough to reflect the independent risk factor that these inflammatory rheumatic diseases represent. Attempts to correct this have been made with the EULAR guideline that suggested a multiplicative factor of 1.5 , which is applied to whichever cardiac risk score is used for certain RA populations [25]. While the multiplicative factor does not apply to the SLE patient at this time, an annual CV risk assessment is recommended in the 2010 EULAR guidelines for monitoring SLE patients [26].

Rather than focusing on traditional CV risk factors, some rheumatologists may feel that aggressive disease control to suppress inflammation in SLE and IA patients may be pivotal in reducing cardiovascular risk rather than traditional risk factor assessment and reduction [2, 17]. Enough data, however, suggest that both SLE and IA patients have traditional cardiac risk factors that should be modified as they also contribute to future CV events [31, 32].

A dedicated cardiovascular risk reduction clinic for inflammatory rheumatic diseases has been established at our site as a result of this review to address the need for more effective $\mathrm{CV}$ risk stratification and risk reduction. The clinic will also serve to follow a prospective cohort of patients over time and evaluate existing guidelines and possible treatment protocols for patients while considering their DMARD or biologic therapies as well. Other possible solutions already suggested by the EULAR include incorporating a cardiovascular risk scoring system into the annual evaluations of IA and SLE patients and implementing aggressive risk reduction in the rheumatology clinic at that time. The results of this study suggest that currently, this strategy is ineffective, at least at our site. Efforts must be placed in educating both primary care physicians and rheumatologists on the increased CV risk in these patients and ensuring $\mathrm{CV}$ risk assessment is truly standard of care. At this time, simple measures such as traditional risk factor reduction and aggressive disease modification can be instituted until more clear data arise from prospective, large-scale cohorts on the best way to treat the increased $\mathrm{CV}$ risk in these patients with inflammatory rheumatic diseases.

Acknowledgment The authors would like to thank the rheumatologists at the Division of Rheumatology, University of Alberta for granting permission to review their patients for this study.

Disclosures None

\section{References}

1. Shoenfeld Y, Gerli R, Doria A et al (2005) Accelerated atherosclerosis in autoimmune rheumatic diseases. Circulation 112:3337-3347

2. Nurmohamed MT (2009) Cardiovascular risk in rheumatoid arthritis. Autoimmun Rev 8:663-667

3. Bartoloni Bocci E, Luccioli F, Angrisani C, Moscatelli S, Alunno A, Gerli R (2007) Accelerated atherosclerosis in systemic lupus erythematosus and other connective tissue diseases. Expert Rev Clin Immunol 3:531-541

4. Meune C, Touzé E, Trinquart L, Allanore Y (2009) Trends in cardiovascular mortality in patients with rheumatoid arthritis over 50 years: a systematic review and meta-analysis of cohort studies. Rheumatol (Oxford) 48:1309-1313

5. Khan F, Galarraga B, Belch JJF (2010) The role of endothelial function and its assessment in rheumatoid arthritis. Nat Rev Rheumatol 6:253-261

6. Solomon DH, Karlson EW, Rimm EB et al (2003) Cardiovascular morbidity and mortality in women diagnosed with rheumatoid arthritis. Circulation 107:1303-1307

7. Avouac J, Allanore Y (2008) Cardiovascular risk in rheumatoid arthritis: effects of anti-TNF drugs. Expert Opin Pharmacother 9:1121-1128

8. Gabriel SE, Crowson CS, Kremers HM et al (2003) Survival in rheumatoid arthritis: a population-based analysis of trends over 40 years. Arthritis Rheum 48:54-58

9. Kroot EJ, van Leeuwen MA, van Rijswijk MH et al (2000) No increased mortality in patients with rheumatoid arthritis: up to 10 years of follow up from disease onset. Ann Rheum Dis 59:954-958

10. Symmons DPM, Jones MA, Scott DL, Prior P (1998) Long term mortality outcome in patients with rheumatoid arthritis: early presenters continue to do well. J Rheumatol 25:5 
11. Reilly PA, Cosh JA, Maddison PJ, Silman AJ (1990) Mortality and survival in rheumatoid arthritis: a 25 year prospective study of 100 patients. Ann Rheum Dis 49:3

12. Kremers HM, Crowson CS, Therneau TM, Roger VL, Gabriel SE (2008) High ten-year risk of cardiovascular disease in newly diagnosed rheumatoid arthritis patients: a population-based cohort study. Arthritis Rheum 58:2268-2274

13. Manzi S, Meilahn EN, Rairie JE et al (1997) Age-specific incidence rates of myocardial infarction and angina in women with systemic lupus erythematosus: comparison with the Framingham Study. Am J Epidemiol 145:408-415

14. Ward MM (1999) Premature morbidity from cardiovascular and cerebrovascular diseases in women with systemic lupus erythematosus. Arthritis Rheum 42:338-346

15. Fischer LM, Schlienger RG, Matter C, Jick H, Meier CR (2004) Effect of rheumatoid arthritis or systemic lupus erythematosus on the risk of first-time acute myocardial infarction. Am J Cardiol 93:198-2000

16. Hak AE, Karlson EW, Feskanich D, Stampfer MJ, Costenbader $\mathrm{KH}$ (2009) Systemic lupus erythematosus and the risk of cardiovascular disease: results from the nurses' health study. Arthritis Rheum 61(10):1396-1402

17. Bartoloni E, Alunno A, Bistoni O, Gerli R (2011) Cardiovascular risk in rheumatoid arthritis and systemic autoimmune rheumatic disorders: a suggested model of preventive strategy. Clinic Rev Allerg Immunol (in press)

18. de Groot L, Posthumus MD, Kallenberg CGM, Bijl M (2010) Risk factors and early detection of atherosclerosis in rheumatoid arthritis. Eur J Clin Investig 40:835-842

19. Ozbalkan Z, Efe C, Cesur M et al (2010) An update on the relationships between rheumatoid arthritis and atherosclerosis. Atherosclerosis 212:377-382

20. Johnsen SP, Larsson H, Tarone RE et al (2005) Risk of hospitalization for myocardial infarction among users of rofecoxib, celecoxib, and other NSAIDs: a population-based casecontrol study. Arch Intern Med 165:978-984

21. del Rincón I, O’Leary DH, Haas RW, Escalante A (2004) Effect of glucocorticoids on the arteries in rheumatoid arthritis. Arthritis Rheum 50:3813-3822
22. García-Gómez C, Nolla JM, Valverde J, Narváez J, Corbella E, Pintó X (2008) High HDL-cholesterol in women with rheumatoid arthritis on low-dose glucocorticoid therapy. Eur J Clin Investig 38:686-692

23. van Ede AE, Laan RFJM, Blom HJ et al (2002) Homocysteine and folate status in methotrexate-treated patients with rheumatoid arthritis. Rheumatol Oxf 41:658-665

24. Roubenoff R, Dellaripa P, Nadeau MR et al (1997) Abnormal homocysteine metabolism in rheumatoid arthritis. Arthritis Rheum 40:718-722

25. Peters MJ, Symmons DP, McCarey D et al (2010) EULAR evidence-based recommendations for cardiovascular risk management in patients with rheumatoid arthritis and other forms of inflammatory arthritis. Ann Rheum Dis 69:325-331

26. Mosca M, Tani C, Aringer $M$ et al (2010) European League Against Rheumatism recommendations for monitoring patients with systemic lupus erythematosus in clinical practice and in observational studies. Ann Rheum Dis 69:1269-1274

27. D'Agostino RB, Ramachandran S, Pencina MJ, The Framingham Heart Study et al (2008) General cardiovascular risk profile for use in primary care. Circulation 117:743-753

28. Hochberg MC (1997) Updating the American College of Rheumatology revised criteria for the classification of systemic lupus erythematosus. Arthritis Rheum 40(9):1725

29. Genest J, McPherson R, Frohlich J et al (2009) Canadian Cardiovascular Society/Canadian guidelines for the diagnosis and treatment of dyslipiedmia and prevention of cardiovascular disease in the adult -2009 recommendations. Can J Cardiol 25 (10):567-579

30. Bell C, Rowe I (2010) The recognition and assessment of cardiovascular risk in people with rheumatoid arthritis in primary care: a questionnaire-based study of general practitioners. Musculoskeletal Care (in press)

31. Brady SR, de Courten B, Reid CM, Cicuttini FM, de Courten MP, Liew D (2009) The role of traditional cardiovascualr risk factors among patients with rheumatoid arthritis. J Rheumatol 36(1):34-40

32. Nikpour M, Urowitz MB, Gladman DD (2009) Epidemiology of atherosclerosis in systemic lupus erythematosus. Curr Rheumatol Rep 11:248-254 